\title{
LINEAR DIFFERENTIAL EQUATIONS WITH ANALYTIC COEFFICIENTS OF $[p, q]$-ORDER IN THE UNIT DISC
}

\author{
ZINELAÂBIDINE LATREUCH AND BENHARRAT BELAÏDI
}

\begin{abstract}
In this paper, we investigate the complex higher order linear differential equations in which the coefficients are analytic functions in the unit disc of $[p, q]$-order. We obtain several theorems about the growth and oscillation of solutions of differential equations.
\end{abstract}

\section{INTRODUCTION AND MAIN RESULTS}

Nevanlinna theory has appeared to be a powerful tool in the field of complex differential equations. For an introduction to the theory of differential equations in the complex plane by using the Nevanlinna theory see [22] . Active research in this field was started by H. Wittich [27] and his students in the 1950's and 1960's. After that many authors have investigated the complex differential equation

$$
f^{(k)}+A_{k-1}(z) f^{(k-1)}+\cdots+A_{1}(z) f^{\prime}+A_{0}(z) f=0
$$

and achieved many valuable results when the coefficients $A_{0}(z), \ldots, A_{k-1}(z)$ are entire functions of finite order $[9,13,18,22]$. L. G. Bernal, L. Kinnunen, $\mathrm{J}$. Tu and $\mathrm{T}$. Long investigated the growth of solutions of (1.1) individually when the coefficients are entire functions of finite iterated order (see $[4,21,26]$. The properties of the growth of (1.1) also have been studied by J. Heittokangas, T. B. Cao and B. Belaïdi when the coefficients are analytic functions in the unit disc $\Delta=\{z:|z|<1\}$ (see $[2,3,5-8,11,15,17,23]$ ). After that A. El Farissi, B. Belaïdi and Z. Latreuch generalized the results of T. B. Cao and investigated the growth of differential polynomial generated by solutions of second order differential equations in the unit disc see Theorem C). In [19], [20] O. P. Juneja and his co-authors investigated some properties of entire functions of $[\mathrm{p}, \mathrm{q}]$-order, and obtain some results. J. Liu, J. Tu and

2010 Mathematics Subject Classification. 34M10, 30D35.

Key words and phrases. Linear differential equations, $[p, q]$-order, analytic function, hyper-order, exponent of convergence of the sequence of distinct zeros. 
L. Z. Shi applied the concepts of entire functions of $[p, q]$-order to investigate the complex differential equation (1.1) (see [24]).

In this paper, we assume that the reader is familiar with the fundamental results and the standard notation of the Nevanlinna's theory on the complex plane and in the unit disc $\Delta=\{z \in \mathbb{C}:|z|<1\}$, see $[12,14,15,25,27]$. In addition, we will use $\lambda(f)$ and $\bar{\lambda}(f)$ to denote respectively the exponents of convergence of the zero-sequence and the sequence of distinct zeros of a meromorphic function $f$ in $\Delta, \rho(f)$ to denote the order, $\rho_{2}(f)$ to denote the hyper-order and $\tau(f)$ to denote the type of $f$. See $[5,11,15,23,25]$ for notations and definitions.

In the following, we will give similar definitions as in $[19,20]$ for analytic and meromorphic functions of $[p, q]$-order, $[p, q]$-type and $[p, q]$-exponent of convergence of the zero sequence in the unit disc.

Definition A. Let $p \geq q \geq 1$ be integers, and let $f$ be a meromorphic function in $\Delta$, the $[p, q]$-order of $f(z)$ is defined by

$$
\rho_{[p, q]}(f)=\limsup _{r \rightarrow 1^{-}} \frac{\log _{p}^{+} T(r, f)}{\log _{q} \frac{1}{1-r}} .
$$

For an analytic function $f$ in $\Delta$, we also define

$$
\rho_{M,[p, q]}(f)=\limsup _{r \rightarrow 1^{-}} \frac{\log _{p+1}^{+} M(r, f)}{\log _{q} \frac{1}{1-r}} .
$$

Remark 1.1. It is easy to see that $0 \leq \rho_{[p, q]}(f) \leq \infty\left(0 \leq \rho_{M,[p, q]}(f) \leq \infty\right)$, for any $p \geq q \geq 1$. By Definition A we have that $\rho_{[1,1]}=\rho(f)\left(\rho_{M,[1,1]}=\right.$ $\left.\rho_{M}(f)\right)$ and $\rho_{[2,1]}=\rho_{2}(f)\left(\rho_{M,[2,1]}=\rho_{M, 2}(f)\right)$.

For the relationship between $\rho_{[p, q]}(f)$ and $\rho_{M,[p, q]}(f)$ we have the following double inequality:

Proposition 1.1. [3] Let $p \geq q \geq 1$ be integers, and let $f$ be an analytic function in $\Delta$ of $[p, q]$-order.

(i) If $p=q$, then

$$
\rho_{[p, q]}(f) \leq \rho_{M,[p, q]}(f) \leq \rho_{[p, q]}(f)+1 .
$$

(ii) If $p>q$, then

$$
\rho_{[p, q]}(f)=\rho_{M,[p, q]}(f) .
$$

Definition B. Let $p \geq q \geq 1$ be integers. The $[p, q]$-type of a meromorphic function $f(z)$ in $\Delta$ of $[p, q]$-order $\rho(0<\rho<\infty)$ is defined by

$$
\tau_{[p, q]}(f)=\limsup _{r \rightarrow 1^{-}} \frac{\log _{p-1}^{+} T(r, f)}{\left(\log _{q-1} \frac{1}{1-r}\right)^{\rho}} .
$$


Definition C. Let $p \geq q \geq 1$ be integers. The $[p, q]$-exponent of convergence of the zero sequence of a meromorphic function $f(z)$ in $\Delta$ is defined by

$$
\lambda_{[p, q]}(f)=\limsup _{r \rightarrow 1^{-}} \frac{\log _{p}^{+} N\left(r, \frac{1}{f}\right)}{\log _{q} \frac{1}{1-r}} .
$$

Similarly, the $[p, q]$-exponent of convergence of the sequence of distinct zeros of $f(z)$ is defined by

$$
\bar{\lambda}_{[p, q]}(f)=\limsup _{r \rightarrow 1^{-}} \frac{\log _{p}^{+} \bar{N}\left(r, \frac{1}{f}\right)}{\log _{q} \frac{1}{1-r}} .
$$

In [6], Cao and Yi have investigated the growth, hyper-order and fixed points of solutions of (1.1) and have obtained the following results.

Theorem A. [6] Let $A_{0}(z), \ldots, A_{k-1}(z)$ be the coefficients of (1.1) analytic in $\Delta$. If $\max \left\{\rho\left(A_{j}\right): j=1, \ldots, k-1\right\}<\rho\left(A_{0}\right)$, then $\rho\left(A_{0}\right) \leq \rho_{2}(f) \leq \alpha_{M}$ holds for all solutions $f \not \equiv 0$ of $(1.1)$, where $\alpha_{M}=\max \left\{\rho_{M}\left(A_{j}\right): j=\right.$ $0, \ldots, k-1\}$.

Theorem B. [6] Under the hypotheses of Theorem A, if $\rho_{2}\left(A_{j}\right)<\infty(j=$ $0, \ldots, k-1)$, then every solution $f \not \equiv 0$ of $(1.1)$ satisfies $\bar{\lambda}_{2}(f-z)=\rho_{2}(f)$.

Recently in [11], the authors have investigated the growth and oscillation of differential polynomials generated by solutions of second order differential equations and obtained the following result.

Theorem C. [11] Let $A_{0}(z), A_{1}(z), d_{0}, d_{1}, d_{2}$ be analytic functions in $\Delta$ such that

$$
\begin{gathered}
\max \left\{\rho\left(A_{1}\right), \rho\left(d_{j}\right)(j=0,1,2)\right\}<\rho\left(A_{0}\right)=\rho(0<\rho<\infty), \\
\tau\left(A_{0}\right)=\tau(0<\tau<\infty),
\end{gathered}
$$

and let $\varphi \not \equiv 0$ be an analytic function in $\Delta$ with $\rho(\varphi)<\infty$. If $f \not \equiv 0$ is a solution of the equation

$$
f^{\prime \prime}+A_{1}(z) f^{\prime}+A_{0}(z) f=0,
$$

then the differential polynomial $g_{f}=d_{2} f^{\prime \prime}+d_{1} f^{\prime}+d_{0} f$ satisfies

$$
\begin{gathered}
\bar{\lambda}\left(g_{f}-\varphi\right)=\lambda\left(g_{f}-\varphi\right)=\rho\left(g_{f}\right)=\rho(f)=\infty, \\
\alpha_{M} \geq \bar{\lambda}_{2}\left(g_{f}-\varphi\right)=\lambda_{2}\left(g_{f}-\varphi\right)=\rho_{2}\left(g_{f}\right)=\rho_{2}(f) \geq \rho\left(A_{0}\right),
\end{gathered}
$$

where $\alpha_{M}=\max \left\{\rho_{M}\left(A_{j}\right): j=0,1\right\}$. 
The aim of this paper is to make use the concepts of analytic functions in the unit disc of $[p, q]$-order and $[p, q]$-type to obtain several theorems about the growth and oscillation of solutions of differential equations (1.1) and (1.2).

Theorem 1.1. Let $p \geq q \geq 1$ be integers, and let $A_{j}(z)(j=0, \ldots, k-1)$ be analytic functions in $\Delta$ satisfying

$$
\max \left\{\rho_{[p, q]}\left(A_{j}\right): j=1, \ldots, k-1\right\}<\rho_{[p, q]}\left(A_{0}\right) .
$$

If $f \not \equiv 0$ is a solution of $(1.1)$, then $\rho_{[p, q]}(f)=\infty$ and

$$
\rho_{[p, q]}\left(A_{0}\right) \leq \rho_{[p+1, q]}(f) \leq \max \left\{\rho_{M,[p, q]}\left(A_{j}\right): j=0, \ldots, k-1\right\} .
$$

Furthermore, if $p>q$, then

$$
\rho_{[p+1, q]}(f)=\rho_{[p, q]}\left(A_{0}\right) .
$$

Theorem 1.2. Let $p \geq q \geq 1$ be integers. Suppose that $A_{0}(z), \ldots, A_{k-1}(z)$ satisfy the hypotheses of Theorem 1.1, and let $\varphi \not \equiv 0$ be an analytic function in $\Delta$ such that $\rho_{[p, q]}(\varphi)<\infty$. Then every solution $f \not \equiv 0$ of (1.1) satisfies

$$
\bar{\lambda}_{[p, q]}(f-\varphi)=\lambda_{[p, q]}(f-\varphi)=\rho_{[p, q]}(f)=\infty
$$

and

$$
\bar{\lambda}_{[p+1, q]}(f-\varphi)=\lambda_{[p+1, q]}(f-\varphi)=\rho_{[p+1, q]}(f) .
$$

Theorem 1.3. Let $p \geq q \geq 1$ be integers, and let $A_{0}(z), A_{1}(z)$ be analytic functions in $\Delta$ such that $\rho_{[p, q]}\left(A_{1}\right)<\rho_{[p, q]}\left(A_{0}\right)=\rho(0<\rho<\infty)$ and $\tau_{[p, q]}\left(A_{0}\right)=\tau(0<\tau<\infty)$. Let $d_{0}, d_{1}, d_{2}$ be analytic functions in $\Delta$ that do not all vanish identically such that

$$
\max \left\{\rho_{[p, q]}\left(d_{j}\right): j=0,1,2\right\}<\rho_{[p, q]}\left(A_{0}\right) .
$$

If $f \not \equiv 0$ is a solution of $(1.2)$, then the differential polynomial $g_{f}=d_{2} f^{\prime \prime}+$ $d_{1} f^{\prime}+d_{0} f$ satisfies

$$
\rho_{[p, q]}\left(g_{f}\right)=\rho_{[p, q]}(f)=\infty
$$

and

$$
\rho_{[p+1, q]}\left(g_{f}\right)=\rho_{[p+1, q]}(f) .
$$

Theorem 1.4. Let $p \geq q \geq 1$ be integers, and let $A_{0}(z), A_{1}(z)$ be analytic functions in $\Delta$ such that $\rho_{[p, q]}\left(A_{1}\right)<\rho_{[p, q]}\left(A_{0}\right)=\rho(0<\rho<\infty)$ and $\tau_{[p, q]}\left(A_{0}\right)=\tau(0<\tau<\infty)$. Let $d_{0}, d_{1}, d_{2}$ be analytic functions in $\Delta$ that do not all vanish identically such that

$$
\max \left\{\rho_{[p, q]}\left(d_{j}\right): j=0,1,2\right\}<\rho_{[p, q]}\left(A_{1}\right),
$$


and let $\varphi \not \equiv 0$ be an analytic function in $\Delta$ such that $\rho_{[p, q]}(\varphi)<\infty$. If $f \not \equiv 0$ is a solution of (1.2), then the differential polynomial $g_{f}=d_{2} f^{\prime \prime}+d_{1} f^{\prime}+d_{0} f$ satisfies

$$
\bar{\lambda}_{[p, q]}\left(g_{f}-\varphi\right)=\lambda_{[p, q]}\left(g_{f}-\varphi\right)=\rho_{[p, q]}(f)=\infty
$$

and

$$
\bar{\lambda}_{[p+1, q]}\left(g_{f}-\varphi\right)=\lambda_{[p+1, q]}\left(g_{f}-\varphi\right)=\rho_{[p+1, q]}(f) .
$$

2. Some PRELiminary LEMmaS

Lemma 2.1. $[12,14,15]$ Let $f$ be a meromorphic function in the unit disc and let $k \in \mathbb{N}$. Then

$$
m\left(r, \frac{f^{(k)}}{f}\right)=S(r, f),
$$

where $S(r, f)=O\left(\log ^{+} T(r, f)+\log \left(\frac{1}{1-r}\right)\right)$, possibly outside a set $E_{0} \subset$ $[0,1)$ with $\int_{E_{0}} \frac{d r}{1-r}<\infty$.

Lemma 2.2. [1] Let $g:(0,1) \rightarrow \mathbb{R}$ and $h:(0,1) \rightarrow \mathbb{R}$ be monotone increasing functions such that $g(r) \leq h(r)$ holds outside of an exceptional set $E_{1} \subset[0,1)$ for which $\int_{E_{1}} \frac{d r}{1-r}<\infty$. Then there exists a constant $d \in(0,1)$ such that if $s(r)=1-d(1-r)$, then $g(r) \leq h(s(r))$ for all $r \in[0,1)$.

Lemma 2.3. Let $p \geq q \geq 1$ be integers. Let $A_{j}(j=0, \ldots, k-1), F \not \equiv 0$ be analytic functions in $\Delta$, and let $f(z)$ be a solution of the differential equation

$$
f^{(k)}+A_{k-1}(z) f^{(k-1)}+\cdots+A_{1}(z) f^{\prime}+A_{0}(z) f=F
$$

satisfying $\max \left\{\rho_{[p, q]}\left(A_{j}\right)(j=0, \ldots, k-1), \rho_{[p, q]}(F)\right\}<\rho_{[p, q]}(f)=\rho \leq$ $\infty$. Then we have

$$
\bar{\lambda}_{[p, q]}(f)=\lambda_{[p, q]}(f)=\rho_{[p, q]}(f)
$$

and

$$
\bar{\lambda}_{[p+1, q]}(f)=\lambda_{[p+1, q]}(f)=\rho_{[p+1, q]}(f) .
$$

Proof. We prove the lemma when $\rho_{[p, q]}(f)=\rho<\infty$. For $\rho_{[p, q]}(f)=\rho=\infty$ we use a similar proof. From the equation (2.1) we get

$$
\frac{1}{f}=\frac{1}{F}\left(\frac{f^{(k)}}{f}+A_{k-1} \frac{f^{(k-1)}}{f}+\cdots+A_{1} \frac{f^{\prime}}{f}+A_{0}\right) .
$$


If $f$ has a zero at $z_{0} \in \Delta$ of order $\alpha(\alpha>k)$, then we get from (2.1) that $F$ has a zero at $z_{0}$ of order at least $\alpha-k$. Hence we have

$$
n\left(r, \frac{1}{f}\right) \leq k \bar{n}\left(r, \frac{1}{f}\right)+n\left(r, \frac{1}{F}\right)
$$

and

$$
N\left(r, \frac{1}{f}\right) \leq k \bar{N}\left(r, \frac{1}{f}\right)+N\left(r, \frac{1}{F}\right) .
$$

By the Lemma 2.1 and (2.2), we have

$$
m\left(r, \frac{1}{f}\right) \leq m\left(r, \frac{1}{F}\right)+\sum_{j=0}^{k-1} m\left(r, A_{j}\right)+O\left(\log ^{+} T(r, f)+\log \frac{1}{1-r}\right)
$$

holds for all $|z|=r \notin E_{0}$, where $E_{0}$ is a subset of [0,1[ with $\int_{E_{0}} \frac{d r}{1-r}<\infty$. By (2.3) and (2.4) we get for all $|z|=r \notin E_{0}$

$$
\begin{gathered}
T(r, f)=T\left(r, \frac{1}{f}\right)+O(1) \\
\leq k \bar{N}\left(r, \frac{1}{f}\right)+T(r, F)+\sum_{j=0}^{k-1} T\left(r, A_{j}\right)+O\left(\log ^{+} T(r, f)+\log \frac{1}{1-r}\right) .
\end{gathered}
$$

Set $\max \left\{\rho_{[p, q]}\left(A_{j}\right)(j=0, \ldots, k-1), \rho_{[p, q]}(F)\right\}=\beta<\rho_{[p, q]}(f)=\rho$. For any given $(0<2 \varepsilon<\rho-\beta)$ and $r \rightarrow 1^{-}$, we have

$$
\begin{gathered}
O\left(\log ^{+} T(r, f)+\log \frac{1}{1-r}\right) \leq \frac{1}{2} T(r, f) \\
\sum_{j=0}^{k-1} T\left(r, A_{j}\right)+T(r, F) \leq(k+1) \exp _{p}\left\{(\beta+\varepsilon) \log _{q}\left(\frac{1}{1-r}\right)\right\} \\
=o(1) \exp _{p}\left\{(\rho-\varepsilon) \log _{q}\left(\frac{1}{1-r}\right)\right\} .
\end{gathered}
$$

Thus, by $(2.5)-(2.7)$, we have

$$
T(r, f) \leq 2 k \bar{N}\left(r, \frac{1}{f}\right)+o(1) \exp _{p}\left\{(\rho-\varepsilon) \log _{q}\left(\frac{1}{1-r}\right)\right\}\left(r \notin E_{0}\right) .
$$

By using Lemma 2.2, we obtain from (2.8) that

$$
\bar{\lambda}_{[p, q]}(f)=\lambda_{[p, q]}(f)=\rho_{[p, q]}(f)=\rho
$$

and

$$
\bar{\lambda}_{[p+1, q]}(f)=\lambda_{[p+1, q]}(f)=\rho_{[p+1, q]}(f) .
$$


By using Theorem 5.1 in [16], we easily obtain the following lemma. For details see [3] .

Lemma 2.4. Let $p \geq q \geq 1$ be integers. If $A_{0}(z), \ldots, A_{k-1}(z)$ are analytic functions of $[p, q]$-order in the unit disc $\Delta$, then every solution $f \not \equiv 0$ of (1.1) satisfies

$$
\rho_{[p+1, q]}(f)=\rho_{M,[p+1, q]}(f) \leq \max \left\{\rho_{M,[p, q]}\left(A_{j}\right): j=0,1, \ldots, k-1\right\} .
$$

The proofs of the following two lemmas are essentially the same as in the corresponding results for the usual order and type. For details, see Chapter 2 of the book by Goldberg-Ostrovskii [12]. So, we omit the proofs.

Lemma 2.5. Let $p \geq q \geq 1$ be integers, and let $f$ and $g$ be meromorphic functions of $[p, q]$-order in $\Delta$. Then we have

$$
\rho_{[p, q]}(f+g) \leq \max \left\{\rho_{[p, q]}(f), \rho_{[p, q]}(g)\right\}
$$

and

$$
\rho_{[p, q]}(f g) \leq \max \left\{\rho_{[p, q]}(f), \rho_{[p, q]}(g)\right\} .
$$

Furthermore, if $\rho_{[p, q]}(f)>\rho_{[p, q]}(g)$, then we obtain

$$
\rho_{[p, q]}(f+g)=\rho_{[p, q]}(f g)=\rho_{[p, q]}(f) .
$$

Lemma 2.6. Let $p \geq q \geq 1$ be integers, and let $f$ and $g$ be meromorphic functions of $[p, q]$-order in $\Delta$ such that $0<\rho_{[p, q]}(f), \rho_{[p, q]}(g)<\infty$ and $0<\tau_{[p, q]}(f), \tau_{[p, q]}(g)<\infty$. We have

(i) If $\rho_{[p, q]}(f)>\rho_{[p, q]}(g)$, then

$$
\tau_{[p, q]}(f+g)=\tau_{[p, q]}(f g)=\tau_{[p, q]}(f) .
$$

(ii) If $\rho_{[p, q]}(f)=\rho_{[p, q]}(g)$ and $\tau_{[p, q]}(f) \neq \tau_{[p, q]}(g)$, then

$$
\rho_{[p, q]}(f+g)=\rho_{[p, q]}(f g)=\rho_{[p, q]}(f)=\rho_{[p, q]}(g) .
$$

The following lemma is a simple consequence of Lemma 2.1. For details see [3].

Lemma 2.7. Let $p \geq q \geq 1$ be integers. Let $f$ be a meromorphic function in the unit disc $\Delta$ such that $\rho_{[p, q]}(f)=\rho<\infty$, and let $k \geq 1$ be an integer. Then for any $\varepsilon>0$,

$$
m\left(r, \frac{f^{(k)}}{f}\right)=O\left(\exp _{p-1}\left\{(\rho+\varepsilon) \log _{q}\left(\frac{1}{1-r}\right)\right\}\right)
$$

holds for all $r$ outside a set $E_{2} \subset[0,1)$ with $\int_{E_{2}} \frac{d r}{1-r}<\infty$. 
By using the result of Chuang [10] about the relation between $T\left(r, f^{\prime}\right)$ and $T(r, f)$, we can obtain the following lemma.

Lemma 2.8. Let $p \geq q \geq 1$ be integers, and let $f$ be a meromorphic function of $[p, q]$-order in $\Delta$. Then $\rho_{[p, q]}\left(f^{\prime}\right)=\rho_{[p, q]}(f)$.

\section{Proof of Theorem 1.1}

Denote $\rho_{[p, q]}\left(A_{0}\right)=\rho$ and let $f \not \equiv 0$ be a solution of (1.1). From equation (1.1) we get

$$
A_{0}=-\left(\frac{f^{(k)}}{f}+A_{k-1} \frac{f^{(k-1)}}{f}+\cdots+A_{1} \frac{f^{\prime}}{f}\right),
$$

then, by Lemma 2.1

$$
m\left(r, A_{0}\right) \leq \sum_{j=1}^{k-1} m\left(r, A_{j}\right)+O\left(\log ^{+} T(r, f)+\log \left(\frac{1}{1-r}\right)\right)
$$

holds for all $|z|=r \notin E_{0}$, where $E_{0}$ is a subset of [0,1[ with $\int_{E_{0}} \frac{d r}{1-r}<\infty$. By Definition A, there exists a sequence $\left\{r_{n}^{\prime}\right\}\left(r_{n}^{\prime} \longrightarrow 1^{-}\right)$such that

$$
\lim _{r_{n}^{\prime} \mapsto 1^{-}} \frac{\log _{p}^{+} T\left(r_{n}^{\prime}, A_{0}\right)}{\log _{q} \frac{1}{1-r_{n}^{\prime}}}=\rho .
$$

Set $\int_{E_{0}} \frac{d r}{1-r}:=\log \gamma<\infty$. Since $\int_{r_{n}^{\prime}}^{1-\frac{1-r_{n}^{\prime}}{\gamma+1}} \frac{d r}{1-r}=\log (\gamma+1)$, then there exists a point $r_{n} \in\left[r_{n}^{\prime}, 1-\frac{1-r_{n}^{\prime}}{\gamma+1}\right]-E_{0} \subset[0,1)$. From

$$
\frac{\log _{p}^{+} T\left(r_{n}, A_{0}\right)}{\log _{q} \frac{1}{1-r_{n}}} \geq \frac{\log _{p}^{+} T\left(r_{n}^{\prime}, A_{0}\right)}{\log _{q}\left(\frac{\gamma+1}{1-r_{n}^{\prime}}\right)}=\frac{\log _{p}^{+} T\left(r_{n}^{\prime}, A_{0}\right)}{\log _{q-1}\left[\left(1+\frac{\log (\gamma+1)}{\log \frac{1}{1-r_{n}^{\prime}}}\right) \log \frac{1}{1-r_{n}^{\prime}}\right]},
$$

it follows that

$$
\lim _{r_{n} \rightarrow 1^{-}} \frac{\log _{p}^{+} T\left(r_{n}, A_{0}\right)}{\log _{q} \frac{1}{1-r_{n}}}=\rho .
$$

Set $\max \left\{\rho_{[p, q]}\left(A_{j}\right): j=1, \ldots, k-1\right\}=\beta<\rho_{[p, q]}\left(A_{0}\right)=\rho$. So, for any given $\varepsilon(0<2 \varepsilon<\rho-\beta)$, we have

$$
T\left(r_{n}, A_{0}\right)>\exp _{p}\left\{(\rho-\varepsilon) \log _{q}\left(\frac{1}{1-r_{n}}\right)\right\}
$$


and for $j=1, \ldots, k-1$

$$
T\left(r_{n}, A_{j}\right) \leq \exp _{p}\left\{(\beta+\varepsilon) \log _{q}\left(\frac{1}{1-r_{n}}\right)\right\}
$$

hold for $r_{n} \rightarrow 1^{-}$. By (3.2), (3.3) and (3.4), we get for $r_{n} \rightarrow 1^{-}$

$$
\begin{gathered}
\exp _{p}\left\{(\rho-\varepsilon) \log _{q}\left(\frac{1}{1-r_{n}}\right)\right\} \leq(k-1) \exp _{p}\left\{(\beta+\varepsilon) \log _{q}\left(\frac{1}{1-r_{n}}\right)\right\} \\
+O\left(\log \frac{1}{1-r_{n}} T\left(r_{n}, f\right)\right) .
\end{gathered}
$$

Noting that $\rho-\varepsilon>\beta+\varepsilon$, it follows from (3.5) that for $r_{n} \rightarrow 1^{-}$

$$
(1-o(1)) \exp _{p}\left\{(\rho-\varepsilon) \log _{q}\left(\frac{1}{1-r_{n}}\right)\right\} \leq O\left(\log \frac{1}{1-r_{n}} T\left(r_{n}, f\right)\right) .
$$

Hence, by (3.6) we obtain $\rho_{[p, q]}(f)=\infty$ and

$$
\rho_{[p+1, q]}(f)=\limsup _{r_{n} \rightarrow 1^{-}} \frac{\log _{p+1}^{+} T\left(r_{n}, f\right)}{\log _{q} \frac{1}{1-r_{n}}} \geq \rho-\varepsilon .
$$

Since $\varepsilon>0$ is arbitrary we get from (3.7) that $\rho_{[p+1, q]}(f) \geq \rho=\rho_{[p, q]}\left(A_{0}\right)$. On the other hand, by Lemma 2.4, we have

$$
\rho_{[p+1, q]}(f)=\rho_{M,[p+1, q]}(f) \leq \max \left\{\rho_{M,[p, q]}\left(A_{j}\right): j=0,1, \ldots, k-1\right\} .
$$

It yields

$$
\begin{aligned}
\rho_{[p, q]}\left(A_{0}\right) \leq \rho_{[p+1, q]}(f)=\rho_{M,[p+1, q]}(f) & \\
& \leq \max \left\{\rho_{M,[p, q]}\left(A_{j}\right): j=0,1, \ldots, k-1\right\} .
\end{aligned}
$$

If $p>q$, then we have

$$
\max \left\{\rho_{M,[p, q]}\left(A_{j}\right): j=0,1, \ldots, k-1\right\}=\rho_{[p, q]}\left(A_{0}\right) .
$$

Therefore, we deduce that

$$
\rho_{[p+1, q]}(f)=\rho_{[p, q]}\left(A_{0}\right) .
$$

\section{Proof of Theorem 1.2}

Suppose that $f \not \equiv 0$ is a solution of equation (1.1). Then by Theorem 1.1, we have $\rho_{[p, q]}(f)=\infty$ and

$$
\rho_{[p, q]}\left(A_{0}\right) \leq \rho_{[p+1, q]}(f) \leq \max \left\{\rho_{M,[p, q]}\left(A_{j}\right): j=0, \ldots, k-1\right\} .
$$

Furthermore, if $p>q$, then

$$
\rho_{[p+1, q]}(f)=\rho_{[p, q]}\left(A_{0}\right) .
$$


Set $w=f-\varphi$. Since $\rho_{[p, q]}(\varphi)<\infty$, then by Lemma 2.5, we have $\rho_{[p, q]}(w)=$ $\rho_{[p, q]}(f-\varphi)=\rho_{[p, q]}(f)=\infty$ and $\rho_{[p+1, q]}(w)=\rho_{[p+1, q]}(f-\varphi)=\rho_{[p+1, q]}(f)$. Substituting $f=w+\varphi$ into equation (1.1), we obtain

$$
\begin{aligned}
w^{(k)}+A_{k-1}(z) & w^{(k-1)}+\cdots+A_{0}(z) w \\
& =-\left(\varphi^{(k)}+A_{k-1}(z) \varphi^{(k-1)}+\cdots+A_{0}(z) \varphi\right)=W .
\end{aligned}
$$

Since $\varphi \not \equiv 0$ and $\rho_{[p, q]}(\varphi)<\infty$, then by Theorem 1.1, we have $W \not \equiv$ 0 . Then by Lemma 2.3 , we obtain $\bar{\lambda}_{[p, q]}(w)=\lambda_{[p, q]}(w)=\rho_{[p, q]}(w)=\infty$ and $\bar{\lambda}_{p+1}(w)=\lambda_{p+1}(w)=\rho_{p+1}(w)$, i.e., $\bar{\lambda}_{[p, q]}(f-\varphi)=\lambda_{[p, q]}(f-\varphi)=$ $\rho_{[p, q]}(f)=\infty$ and $\bar{\lambda}_{[p+1, q]}(f-\varphi)=\lambda_{[p+1, q]}(f-\varphi)=\rho_{[p+1, q]}(f)$.

\section{Proof of Theorem 1.3}

Suppose that $f \not \equiv 0$ is a solution of equation (1.2). Then by Theorem 1.1, we have $\rho_{[p, q]}(f)=\infty$ and

$$
\rho_{[p, q]}\left(A_{0}\right) \leq \rho_{[p+1, q]}(f) \leq \max \left\{\rho_{M,[p, q]}\left(A_{j}\right): j=0,1\right\} .
$$

Furthermore, if $p>q$, then

$$
\rho_{[p+1, q]}(f)=\rho_{[p, q]}\left(A_{0}\right) .
$$

Substituting $f^{\prime \prime}=-A_{1} f^{\prime}-A_{0} f$ into $g_{f}$, we get

$$
g_{f}=\left(d_{1}-d_{2} A_{1}\right) f^{\prime}+\left(d_{0}-d_{2} A_{0}\right) f .
$$

Differentiating both sides of equation (5.1) and replacing $f^{\prime \prime}$ with $f^{\prime \prime}=$ $-A_{1} f^{\prime}-A_{0} f$, we obtain

$$
\begin{aligned}
g_{f}^{\prime}=[ & \left.d_{2} A_{1}^{2}-\left(d_{2} A_{1}\right)^{\prime}-d_{1} A_{1}-d_{2} A_{0}+d_{0}+d_{1}^{\prime}\right] f^{\prime} \\
& +\left[d_{2} A_{0} A_{1}-\left(d_{2} A_{0}\right)^{\prime}-d_{1} A_{0}+d_{0}^{\prime}\right] f .
\end{aligned}
$$

Set

$$
\begin{gathered}
\alpha_{1}=d_{1}-d_{2} A_{1}, \quad \alpha_{0}=d_{0}-d_{2} A_{0}, \\
\beta_{1}=d_{2} A_{1}^{2}-\left(d_{2} A_{1}\right)^{\prime}-d_{1} A_{1}-d_{2} A_{0}+d_{0}+d_{1}^{\prime}, \\
\beta_{0}=d_{2} A_{0} A_{1}-\left(d_{2} A_{0}\right)^{\prime}-d_{1} A_{0}+d_{0}^{\prime} .
\end{gathered}
$$

Then, we have

$$
\alpha_{1} f^{\prime}+\alpha_{0} f=g_{f}, \quad \beta_{1} f^{\prime}+\beta_{0} f=g_{f}^{\prime} .
$$

Set

$$
\begin{array}{r}
h=\alpha_{1} \beta_{0}-\alpha_{0} \beta_{1}=\left(d_{1}-d_{2} A_{1}\right)\left(d_{2} A_{0} A_{1}-\left(d_{2} A_{0}\right)^{\prime}-d_{1} A_{0}+d_{0}^{\prime}\right) \\
-\left(d_{0}-d_{2} A_{0}\right)\left(d_{2} A_{1}^{2}-\left(d_{2} A_{1}\right)^{\prime}-d_{1} A_{1}-d_{2} A_{0}+d_{0}+d_{1}^{\prime}\right) .
\end{array}
$$


First we suppose that $d_{2} \not \equiv 0$. Now check all the terms of $h$. Since the term $d_{2}^{2} A_{1}^{2} A_{0}$ is eliminated, by (5.7) we can write

$$
\begin{gathered}
h=-d_{2}^{2} A_{0}^{2}-d_{0} d_{2} A_{1}^{2}+\left(d_{1}^{\prime} d_{2}+2 d_{0} d_{2}-d_{2}^{\prime} d_{1}-d_{1}^{2}\right) A_{0} \\
+\left(d_{2}^{\prime} d_{0}-d_{2} d_{0}^{\prime}+d_{0} d_{1}\right) A_{1}+d_{1} d_{2} A_{0} A_{1} \\
-d_{1} d_{2} A_{0}^{\prime}+d_{0} d_{2} A_{1}^{\prime}+d_{2}^{2} A_{0}^{\prime} A_{1}-d_{2}^{2} A_{0} A_{1}^{\prime}+d_{0}^{\prime} d_{1}-d_{0} d_{1}^{\prime}-d_{0}^{2} .
\end{gathered}
$$

By $d_{2} \not \equiv 0, A_{0} \not \equiv 0$ and Lemmas 2.5-2.6 we have $\rho_{[p, q]}(h)=\rho_{[p, q]}\left(A_{0}\right)$, thus $h \not \equiv 0$. Now suppose $d_{2} \equiv 0, d_{1} \not \equiv 0$, by using a similar reasoning as above we get $h \not \equiv 0$. Finally if $d_{2} \equiv 0, d_{1} \equiv 0$ and $d_{0} \not \equiv 0$, we have $h=-d_{0}^{2} \not \equiv 0$. Hence $h \not \equiv 0$. By $h \not \equiv 0,(5.6)$ and (5.7), we obtain

$$
f=\frac{\alpha_{1} g_{f}^{\prime}-\beta_{1} g_{f}}{h} \text {. }
$$

If $\rho_{[p, q]}\left(g_{f}\right)<\infty$, then by $(5.9)$, Lemma 2.5 and Lemma 2.8 we have $\rho_{[p, q]}(f)<\infty$, and this is a contradiction. Hence $\rho_{[p, q]}\left(g_{f}\right)=\rho_{[p, q]}(f)=\infty$.

Now, we prove that $\rho_{[p+1, q]}\left(g_{f}\right)=\rho_{[p+1, q]}(f)$. By $(5.1)$, Lemma 2.5 and Lemma 2.8, we get $\rho_{[p+1, q]}\left(g_{f}\right) \leq \rho_{[p+1, q]}(f)$ and by $(5.9)$ we have $\rho_{[p+1, q]}(f)$ $\leq \rho_{[p+1, q]}\left(g_{f}\right)$. This yields $\rho_{[p+1, q]}\left(g_{f}\right)=\rho_{[p+1, q]}(f)$.

\section{Proof of Theorem 1.4}

Suppose that $f \not \equiv 0$ is a solution of equation (1.2). Then, by Theorem 1.3, we have $\rho_{[p, q]}\left(g_{f}\right)=\rho_{[p, q]}(f)=\infty$ and $\rho_{[p+1, q]}\left(g_{f}\right)=\rho_{[p+1, q]}(f)$. Set $w(z)=d_{2} f^{\prime \prime}+d_{1} f^{\prime}+d_{0} f-\varphi$. Then, by $\rho_{[p, q]}(\varphi)<\infty$, we have $\rho_{[p, q]}(w)=\rho_{[p, q]}\left(g_{f}\right)=\rho_{[p, q]}(f)=\infty$ and $\rho_{[p+1, q]}(w)=\rho_{[p+1, q]}\left(g_{f}\right)=$ $\rho_{[p+1, q]}(f)$. In order to prove $\bar{\lambda}_{[p, q]}\left(g_{f}-\varphi\right)=\lambda_{[p, q]}\left(g_{f}-\varphi\right)=\rho_{[p, q]}(f)=$ $\infty, \bar{\lambda}_{[p+1, q]}\left(g_{f}-\varphi\right)=\lambda_{[p+1, q]}\left(g_{f}-\varphi\right)=\rho_{[p+1, q]}(f)$, we need to prove only $\bar{\lambda}_{[p, q]}(w)=\lambda_{[p, q]}(w)=\rho_{[p, q]}(f)=\infty, \bar{\lambda}_{[p+1, q]}(w)=\lambda_{[p+1, q]}(w)=\rho_{[p+1, q]}(f)$. Using $g_{f}=w+\varphi$, we get from (5.9)

$$
f=\frac{\alpha_{1} w^{\prime}-\beta_{1} w}{h}+\psi
$$

where

$$
\psi(z)=\frac{\alpha_{1} \varphi^{\prime}-\beta_{1} \varphi}{h} .
$$

Substituting (6.1) into equation (1.2), we obtain

$$
\frac{\alpha_{1}}{h} w^{\prime \prime \prime}+\phi_{2} w^{\prime \prime}+\phi_{1} w^{\prime}+\phi_{0} w=-\left(\psi^{\prime \prime}+A_{1}(z) \psi^{\prime}+A_{0}(z) \psi\right)=A,
$$

where $\phi_{j}(j=0,1,2)$ are meromorphic functions in $\Delta$ with $\rho_{[p, q]}\left(\phi_{j}\right)<\infty$ $(j=0,1,2)$. 
Now, we prove that $\psi(z) \not \equiv 0$. Assume that $\psi(z) \equiv 0$. Then from (6.2), we obtain that

$$
\beta_{1}=\alpha_{1} \frac{\varphi^{\prime}}{\varphi}
$$

Set $\rho_{[p, q]}(\varphi)=\alpha<\infty$. Then, by (6.4) and Lemma 2.7, we have

$$
m\left(r, \beta_{1}\right) \leq m\left(r, \alpha_{1}\right)+O\left(\exp _{p-1}\left\{(\alpha+\varepsilon) \log _{q}\left(\frac{1}{1-r}\right)\right\}\right)
$$

holds for all $r$ outside a set $E_{2} \subset[0,1)$ with $\int_{E_{2}} \frac{d r}{1-r}<\infty$.

(i) If $d_{2} \not \equiv 0$, by Lemma 2.2 and (6.5) we obtain

$$
\rho_{[p, q]}\left(A_{0}\right) \leq \rho_{[p, q]}\left(A_{1}\right) .
$$

This is a contradiction.

(ii) If $d_{2} \equiv 0$ and $d_{1} \not \equiv 0$, by Lemma 2.2 and (6.5) we obtain

$$
\rho_{[p, q]}\left(A_{1}\right) \leq \rho_{[p, q]}\left(d_{1}\right) .
$$

This is a contradiction.

(iii) If $d_{2}=d_{1} \equiv 0$ and $d_{0} \not \equiv 0$, we have by $(6.5)$

$$
\beta_{1}=d_{0}=\alpha_{1} \frac{\varphi^{\prime}}{\varphi} \equiv 0
$$

which is a contradiction. Hence $\psi(z) \not \equiv 0$.

By $\psi(z) \not \equiv 0$ and $\rho_{[p, q]}(\psi)<\infty$, it follows by Theorem 1.1 that $A \not \equiv 0$. Then by $h \not \equiv 0$ and Lemma 2.3, we obtain $\bar{\lambda}_{[p, q]}(w)=\lambda_{[p, q]}(w)=\rho_{[p, q]}(w)=$ $\infty$ and $\bar{\lambda}_{[p+1, q]}(w)=\lambda_{[p+1, q]}(w)=\rho_{[p+1, q]}(w)$, that is, $\bar{\lambda}_{[p, q]}\left(g_{f}-\varphi\right)=$ $\lambda_{[p, q]}\left(g_{f}-\varphi\right)=\rho_{[p, q]}\left(g_{f}\right)=\rho_{[p, q]}(f)=\infty$ and $\bar{\lambda}_{[p+1, q]}\left(g_{f}-\varphi\right)=\lambda_{[p+1, q]}\left(g_{f}\right.$ $-\varphi)=\rho_{[p+1, q]}(f)$.

Acknowledgement. The authors would like to thank the referee for his/her helpful remarks and suggestions to improve the paper.

\section{REFERENCES}

[1] S. Bank, General theorem concerning the growth of solutions of first-order algebraic differential equations, Compositio Math., 25 (1972), 61-70.

[2] B. Belaïdi, Oscillation of fast growing solutions of linear differential equations in the unit disc, Acta Univ. Sapientiae, Mathematica, 2 (1) (2010), 25-38.

[3] B. Belaïdi, Growth of soluitions to linear differential equations with analytic coefficients of $[P, Q]$-order in the unit disc, Electron. J. Diff. Equ., Vol. 2011 (156) (2011), $1-11$.

[4] L. G. Bernal, On growth $k$-order of solutions of a complex homogeneous linear differential equation, Proc. Amer. Math. Soc., 101 (2) (1987), 317-322. 
[5] T. B. Cao and H. X. Yi, On the complex oscillation theory of $f^{\prime \prime}+A f=0$ where $A(z)$ is analytic in the unit disc, Math. Nachr., 282 (6) (2009), 820-831.

[6] T. B. Cao and H. X. Yi, On the complex oscillation theory of of lineare differential equations in the unit disc, Acta Math. Sci., 2008, 28A (6) :1046-1057.

[7] T. B. Cao, The growth, oscillation and fixed points of solutions of complex linear differential equations in the unit disc, J. Math. Anal. Appl., 352 (2) (2009), 739-748.

[8] T. B. Cao and H. X. Yi, The growth of solutions of linear differential equations with coefficients of iterated order in the unit disc, J. Math. Anal. Appl., 319 (2006), 278294.

[9] Z. X. Chen and C. C. Yang, Quantitative estimations on the zeros and growths of entire solutions of linear differential equations, Complex Variables, 42 (2000), 119133.

[10] C. T. Chuang, Sur la comparaison de la croissance d'une fonction méromorphe et de celle de sa dérivée, Bull. Sci. Math., 75 (2) (1951), 171-190.

[11] A. El Farissi, B. Belaïdi and Z. Latreuch, Growth and oscillation of differential polynomials in the unit disc, Electron. J. Diff. Equ., 2010 (87) (2010), 1-7.

[12] A. Goldberg and I. Ostrovskii, Value Distribution of Meromorphic functions, Transl. Math. Monogr., Vol. 236, Amer. Math. Soc., Providence RI, 2008.

[13] G. Gundersen, E. M. Steinbart and S. P. Wang, The possible order of solutions of linear differential equations with polynomial coefficients, Trans. Amer. Math. Soc., 350 (3) (1998), 1225-1247.

[14] W. K. Hayman, Meromorphic Functions, Oxford Mathematical Monographs Clarendon Press, Oxford, 1964

[15] J. Heittokangas, On complex differential equations in the unit disc, Ann. Acad. Sci. Fenn. Math. Diss., 122 (2000), 1-54.

[16] J. Heittokangas, R. Korhonen and J. Rättyä, Growth estimates for solutions of linear complex differential equations, Ann. Acad. Sci. Fenn. Math., 29 (2004), 233-246.

[17] J. Heittokangas, R. Korhonen and J. Rättyä, Fast growing solutions of linear differential equations in the unit disc, Result. Math., 49 (2006), 265-278.

[18] S. Hellerstein, J. Miles and J. Rossi, On the growth of solutions of $f^{\prime \prime}+g f^{\prime}+h f=0$, Trans. Amer. Math. Soc., 324 (1991), 693-706.

[19] O. P. Juneja, G. P. Kapoor and S. K. Bajpai, On the $(p, q)$-order and lower $(p, q)$-order of an entire function, J. Reine Angew. Math. 282 (1976), 53-67.

[20] O. P. Juneja, G. P. Kapoor and S. K. Bajpai, On the $(p, q)$-type and lower $(p, q)$-type of an entire function, J. Reine Angew. Math., 290 (1977), 385-405.

[21] L. Kinnunen, Linear differential equations with solutions of finite iterated order, Southeast Asian Bull. Math., 22 (4) (1998), 4, 385-405.

[22] I. Laine, Nevanlinna Theory and Complex Differential Equations, de Gruyter Studies in Mathematics, 15. Walter de Gruyter \& Co., Berlin, 1993.

[23] Y. Z. Li, On the growth of the solution of two-order differential equations in the unit disc, Pure Appl. Math., 4 (2002), 295-300.

[24] J. Liu, J. Tu and L. Z. Shi, Linear differential equations with entire coefficients of $(p, q)$-order in the complex plane, J. Math. Anal. Appl., 372 (2010), 55-67.

[25] M.Tsuji, Potential Theory in Modern Function Theory, Chelsea, New York, (1975), reprint of the 1959 edition.

[26] J. Tu and T. Long, Oscillation of complex high order linear differential equations with coefficients of finite iterated order, Electron. J. Qual. Theory Differ. Equ., 66 (2009), $1-13$. 
[27] H. Wittich, Neuere Untersuchungen über eindeutige analytishe Funktionen, 2nd Edition, Springer-Verlag, Berlin-Heidelberg-New York, 1968.

(Received: February 7, 2012)

(Revised: May 22, 2012)
Zinelaâbidine Latreuch and Benharrat Belaïdi Department of Mathematics

Laboratory of Pure and Applied Mathematics

University of Mostaganem (UMAB)

B. P. 227

Mostaganem, Algeria

E-mails: z.latreuch@gmail.com

belaidi@univ-mosta.dz 